Automation and Robotics in Construction XII

E.Budny, A.McCrea, K.Szymanski (Editors).

O 1995 IMBiGS. All Pights reserved.

\title{
Development of Marking Robot Working at Building Sites
}

Kouetsu TANAKA*, Makoto KAJITANI ${ }^{* *}$, Chisato KANAMORI ${ }^{* *}$

Hideki ITOH**, Yasunori ABE* and Yoshio TANAKA*

*Shinryo Corporation, Research and Development Center

41, Wadai, Tsukuba, Ibaraki 300-42, Japan

**University of Electro-Communications,

Department of Mechanical and Control Engineering

1-5-1, Chofugaoka, Chofu, Tokyo 182, Japan

\section{Introduction}

Unlike industrial robot working in factories, construction robots must meet more functional requirements because they must handle a variety of complicated jobs at construction sites. For example, interior finishing in a large building often requires robots to place specific marks, drill holes, or mount various fixtures while they are moving on the floor area. We intend to develop a marking robot, which left a specific mark at the fitting position with various apparatus on the ceiling board in large building. In order to accomplish such jobs, the robot must have following two performance;

1)Self-positioning; it must be able to locate itself automatically. In other words, this requires a technique that enables the robot to measure coordinates indicating its position.

2)Marking; it must be able to ink or draw specific marks at the designed position on the ceiling board.

The method to measure self-position proposed in this paper is based on triangulation and it dose not require any exclusive arrangements and but uses existing pillars as reference objects. On the floor of a large building, pillars stand in known positions at intervals of a few meters. They can serve as reference objects. Our system can precisely measure the bearings of the surrounding pillars by using a Laser Range Finder (LRF) and an Angle Measuring Unit (AMU), so that the calculation based on triangulation will be able to give the coordinates of a robot's position. The basic performance of a prototype system was experimentally investigated and the results proved that the system was able to measure the position of $s$ robot with an error of a few millimeters. Next a special drawing equipment similar to a X-Y plotter and a telescopic mechanism which lifted up it were designed and produced for the above second requirement. As the robot moves to the target position is basis on the data obtained from this newly developed self-position measuring system. Our robot accurately got to the desired position and lifted up the drawing equipment. Then it drew various figures. And final accuracy of this marking work was enough to use in 
actual construction sites. In this paper, we show how our robot work concerning selfposition measuring and marking some figures.

\section{Work Process}

This robot consists basically of two units such as self-position measuring system and marking system including mobile system, lifting system and drawing system, as shown in Fig.1. The self-position measuring system can precisely obtain the coordinates of its own location. The mobile system which has two independent driving wheels and a caster wheel carries the other systems and gets to the target point given in advance by human or CAD system. The travel direction and distance are based on data taken at the self-position measuring system. The robot which already understood its way moves by means of dead reckoning depending on rotational angles of independent two driving wheels. On its arrival the self-position measuring system gets is own coordinates again. So if necessary, the robot would slightly move in order to adjust the position. After reconfirming that it accurately stands on designated point, the lifting system raises the drawing system up to the ceiling. Then the specified figures, such as lines, circles, quadrangles, some letters and symbols, are drawn on the ceiling board. This work is continued until the all marks on same floor have been done. The robot is $800 \mathrm{~mm}$ long, $754 \mathrm{~mm}$ width, from $2065 \mathrm{~mm}$ to $3070 \mathrm{~mm}$ variable high and $300 \mathrm{~kg}$ weight. It can work continuously maintaining a speed of $2 \mathrm{~km} / \mathrm{h}$ about 2 hours without charging DC batteries.

\section{Self-position Measurement System}

\subsection{Principle of Measurement}

Suppose that the measuring system can recognize the surrounding pillars and measure two angles $\theta_{21}$ and $\theta_{32}$, whose center is coincident with center of measuring system, by detecting the edges of three pillars (one edge for each pillar) in the manner shown in Fig.2. The coordinates of the center of measuring system are given by the following equations according to the principle of triangulation.

$$
\begin{aligned}
& \mathrm{U}= \mathrm{U}_{2}-\mathrm{AB} /\left(\mathrm{B}^{2}+\mathrm{C}^{2}\right) \\
& \mathrm{V}= \mathrm{V}_{2}-\mathrm{AC} /\left(\mathrm{B}^{2}+\mathrm{C}^{2}\right) \\
& \mathrm{A}=\left\{\left(\mathrm{U}_{1}-\mathrm{U}_{2}\right)\left(\mathrm{U}_{3}-\mathrm{U}_{2}\right)+\left(\mathrm{V}_{1}-\mathrm{V}_{2}\right)\left(\mathrm{V}_{3}-\mathrm{V}_{2}\right)\right\} *\left(\tan \theta_{21}+\tan \theta_{32}\right) \\
&+\left(\left(\mathrm{U}_{3}-\mathrm{U}_{2}\right)\left(\mathrm{V}_{1}-\mathrm{V}_{2}\right)-\left(\mathrm{U}_{1}-\mathrm{U}_{2}\right)\left(\mathrm{V}_{3}-\mathrm{V}_{2}\right)\right\} \cdot\left(1-\tan \theta_{21} \cdot \tan \theta_{32}\right) \\
& \mathrm{B}=\left(\mathrm{V}_{1}-\mathrm{V}_{3}\right) \cdot \tan \theta_{21} \cdot \tan \theta_{32}-\left(\mathrm{U}_{3}-\mathrm{U}_{2}\right) \cdot \tan \theta_{21}\left(\mathrm{U}_{1}-\mathrm{U}_{2}\right) \cdot \tan \theta_{32} \\
& \mathrm{C}=\left(\mathrm{U}_{3}-\mathrm{U}_{1}\right) \cdot \tan \theta_{21} \cdot \tan \theta_{32}-\left(\mathrm{V}_{3}-\mathrm{V}_{2}\right) \cdot \tan \theta_{21}-\left(\mathrm{V}_{1}-\mathrm{V}_{2}\right) * \tan \theta_{32} \\
& \theta_{21}=\theta_{2}-\theta_{1}, \theta_{32}=\theta_{3}-\theta_{2}
\end{aligned}
$$




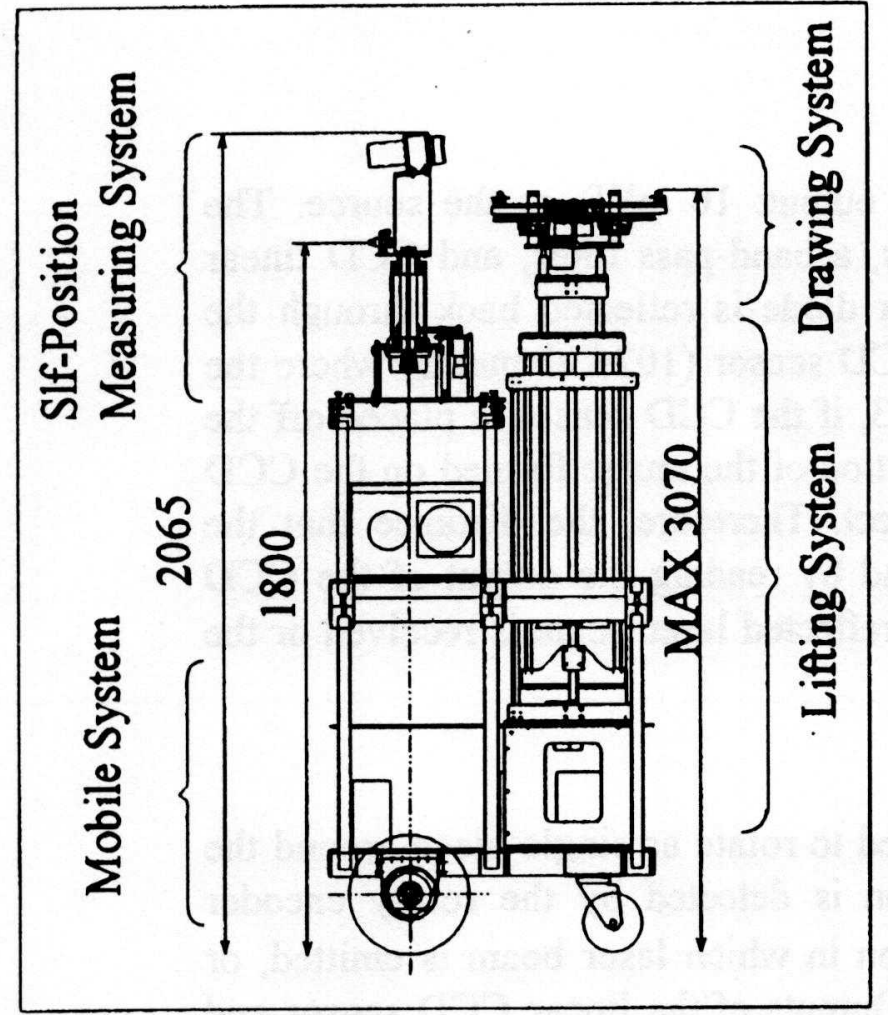

Fig. 1 Marking Robot

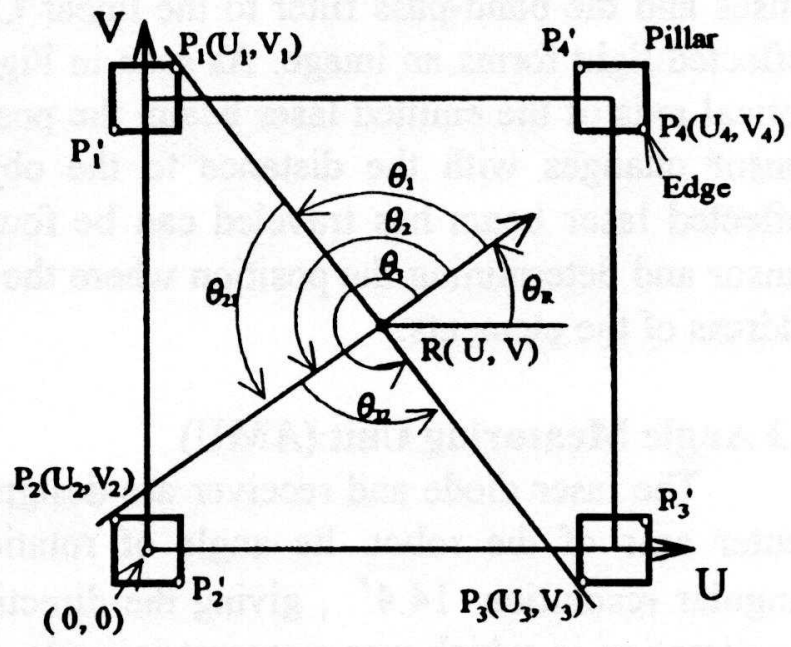

Fig. 2 Measurement Method

where the coordinates of the three edges, $\left(U_{1}, V_{1}\right),\left(U_{2}, V_{2}\right)$ and $\left(U_{3}, V_{3}\right)$, can be known thorough the design specifications.

The robot can actually identify two edges for each of the four pillars, a total of eight edges. Therefore, the position of the robot is determined for each of the eight combinations, resulting in eight measured positions. Then the averaged position is taken as its final position.

\subsection{Laser Range Finder (LRF)}

Fig.3 shows system organization of self-position measuring system. The LRF

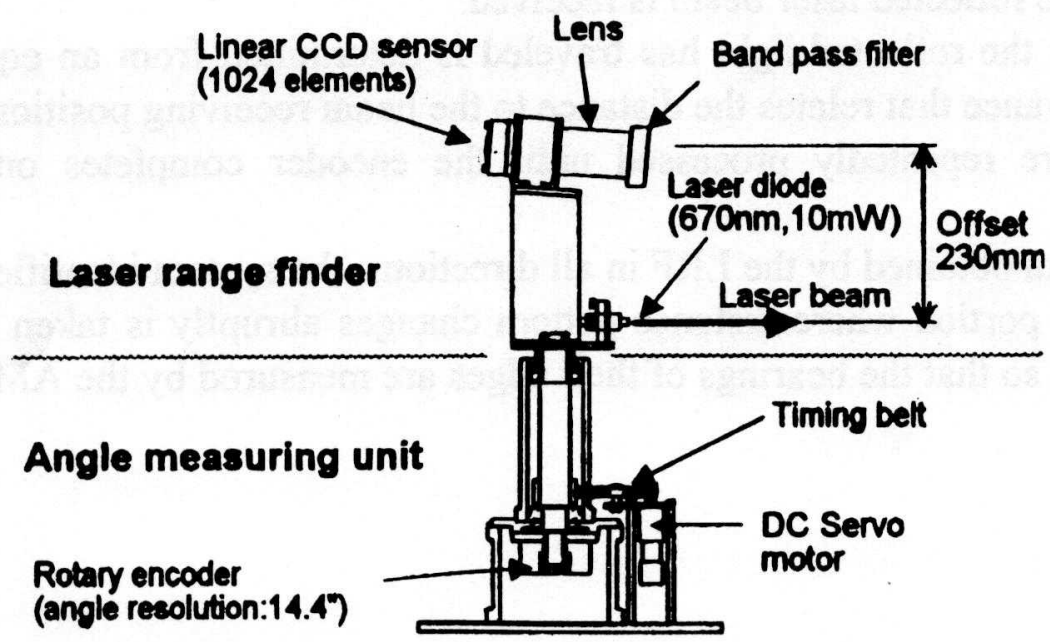

Fig. 3 Self-position Measuring System 
employs a laser diode (wavelength: $670 \mathrm{~nm}$, output: $10 \mathrm{~mW}$ ) as the source. The reflected laser beam receiver consist of lenses, a band-pass filter, and CCD linear sensor. The laser beam emitted from the laser diode is reflected back through the lenses and the band-pass filter to the linear CCD sensor (1024 elements) where the reflected light forms an image. As seen in Fig.3, if the CCD sensor is placed off the optical axis of the emitted laser beam, the position of the image formed on the CCD sensor changes with the distance to the object. Therefore, the distance that the reflected laser beam has traveled can be found by reading the output of the CCD sensor and determining the position where the reflected laser beam is received or the address of the elements.

\subsection{Angle Measuring Unit (AMU)}

The laser diode and receiver are designed to rotate as single piece around the center axis of the robot. Its angle of rotation is detected by the rotary encoder (angular resolution: $14.4^{\prime \prime}$, giving the direction in which laser beam is emitted, or the direction in which measurement is made. Outputs of the linear CCD sensor and the rotary encoder are entered in a computer for processing.

\subsection{Measurement Process}

The steps of the measurement process are as follows:

1) The LRF and the AMU are rotated as a single piece by the DC motor while the robot is at rest.

2) Output data of both the CCD sensor and the rotary encoder are sampled at regular intervals.

3) Address of CCD element, whose output is 50 percent of the maximum output of $1024 \mathrm{CCD}$ elements, $\mathrm{Al}$ and $\mathrm{A} 2$ are found as shown in Fig.4. A1 is the position where the output increase, and $A 2$ is the position where the output decrease.

4) Center address $A 0$, calculated from the relation $A 0=(A 1+A 2) / 2$, is taken as the position where the reflected laser beam is received.

5) The distance that the reflected light has traveled is determined from an equation established in advance that relates the distance to the beam receiving position.

6) Step 2) to 5) are repeatedly processed until the encoder completes one full rotation.

7) From distance data obtained by the LRF in all directions, the system identifies four pillars. Then, the portion where distance pattern changes abruptly is taken as the edge of the pillar, so that the bearings of their edges are measured by the AMU. 
8) From their edges and the known coordinates of the edges, the robot determines its own position according to Eq.(1)-(3).

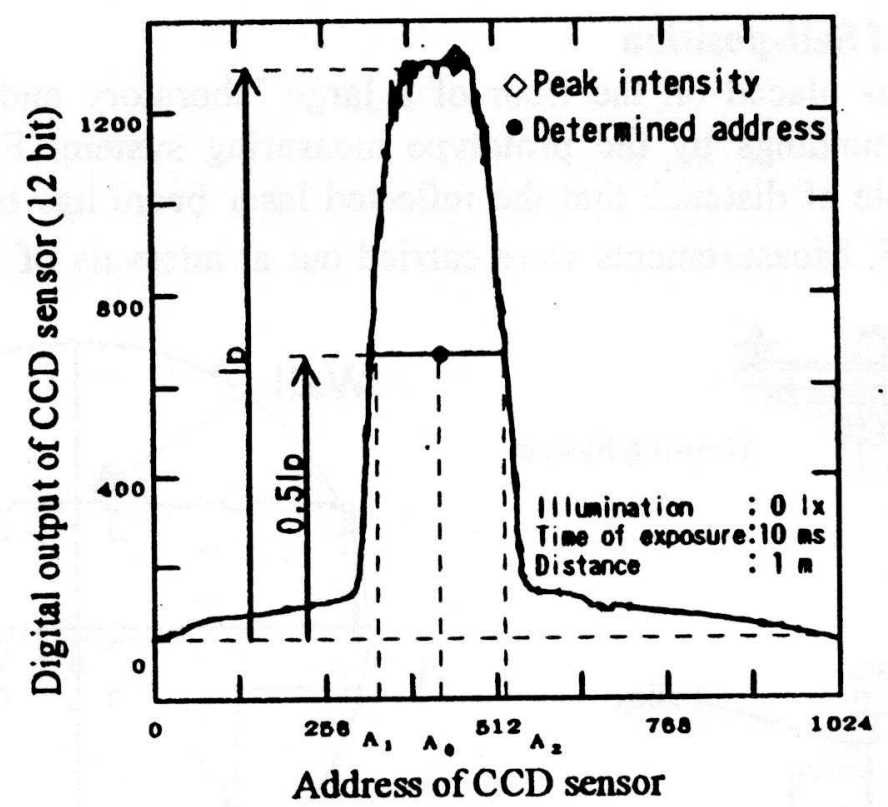

Fig. 4 The output of CCD sensor

\section{Marking}

Fig. 5 shows the marking system which is the other important function in this robot. In order to put a mark on the ceiling board, the system consists of two equipment that are the lifting system and the drawing system. The first one has 1275 $\mathrm{mm}$ stroke to elevate the drawing system and reaches to the ceiling board which is up to $3070 \mathrm{~mm}$ height. Because of most ceiling height in office room is about $2700 \mathrm{~mm}$, the robot can work in a lot of construction sites. It has a telescopic three stages mechanism which is composed mainly of DC servo motor, ball-screw and pulley. The drawing system is lifted up at high speed until the distance between the ceiling and the system is in the range of $150 \mathrm{~mm}$ to $200 \mathrm{~mm}$. Then the photoelectric switch fit on the top of the drawing system turns on, and lift up speed slows down to avoid a shock when hitting the ceiling. As the drawing system touches the ceiling, the touch switches are activated and elevation operation is ended. The second is the drawing system to draw some figures accurately on the ceiling board. These figures are not only various, but also relatively large. Besides the system has to be compact because it is on board the mobile system. Therefore we developed a X-Y-plotter-like equipment whose $x$-axis is divided into two equal parts. The maximum drawing range in $\mathrm{x}$-axis direction is $650 \mathrm{~mm}$ and in $\mathrm{y}$-axis direction is $700 \mathrm{~mm}$. 


\section{Experimental Result}

\subsection{Measurement of Self-position}

The robot was placed on the floor of a large laboratory and measured the distance to its surroundings by the prototype measuring system. Fig. 6 shows a measurement example of distance that the reflected laser beam has traveled, that is the output from LRF. Measurements were carried out at intervals of $14.4^{\prime \prime}$ for the
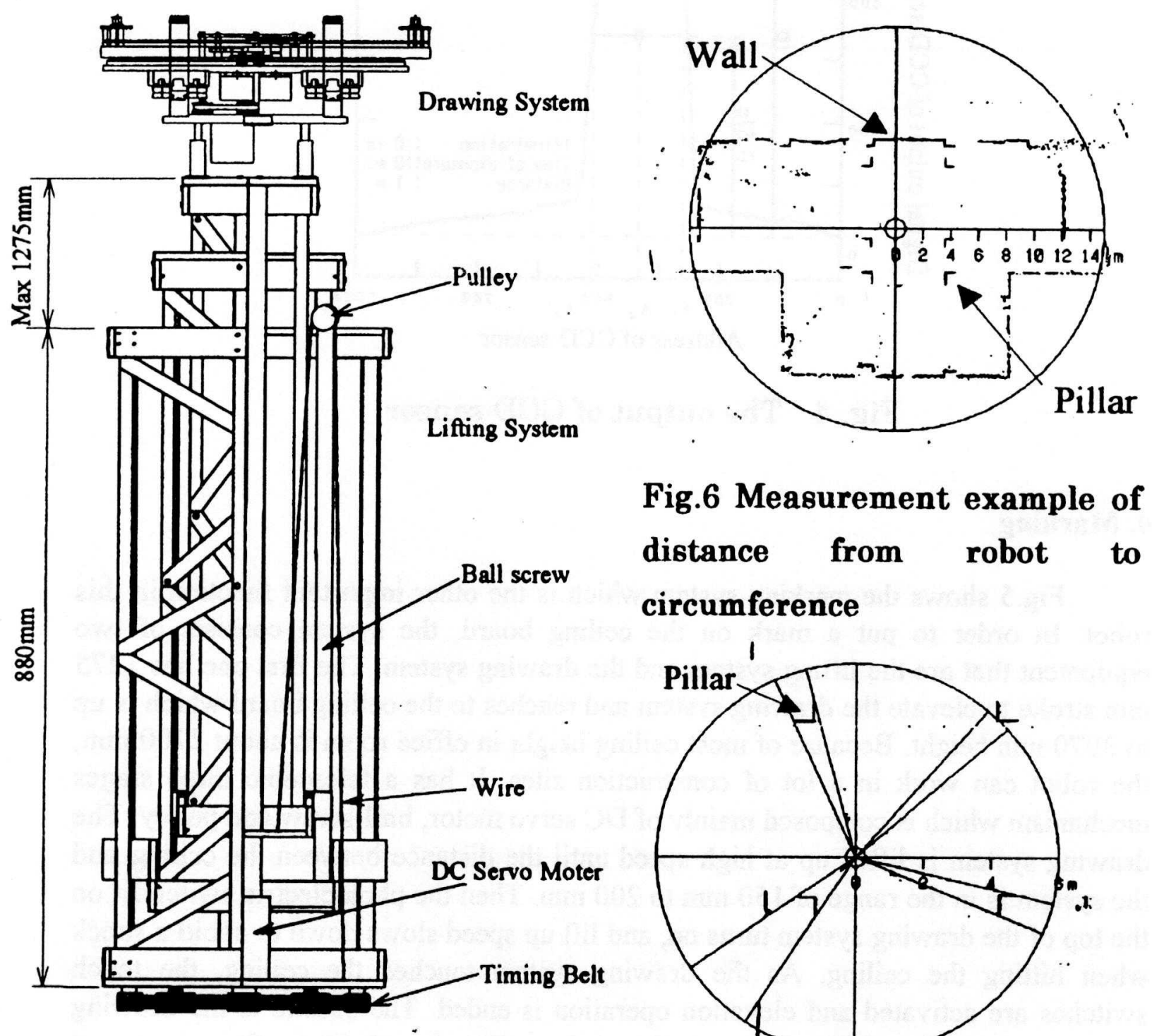

Fig.6 Measurement example of distance from robot to circumference

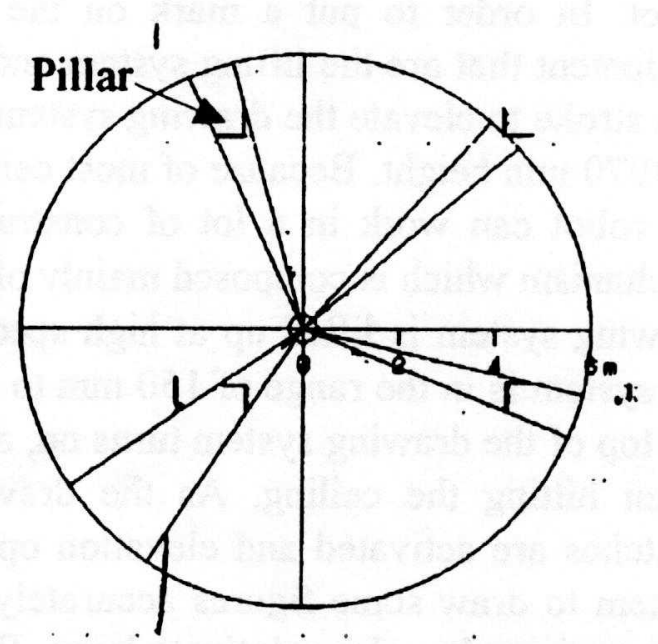

Fig.5 Marking System

Fig.7 Detection example of pillars 
full perimeter of the robot (or 90,000 equal divisions of 360 degrees). The center of the figure corresponds to the center of the robot. As is seen in this figure, the robot can clearly detect pillars, windows and walls located within a range of $15 \mathrm{~m}$.

Fig. 7 shows a detection example of the four pillars $(600 \mathrm{~mm} \times 600 \mathrm{~mm}$ in cross section) which were temporarily set up on the floor of the laboratory. They formed a regular square with sides of $8 \mathrm{~m}$. The robot was placed near the center of the square. As seen in the figure, the robot precisely detected the four pillars and their edges.

Under the same conditions as above, the robot was allowed to move various places and measure its position (See Fig.8). Measurements were repeated 10 or 20 times for each position and each mean value was compared with the measurement value by two transits that were angle measuring instrument. The differences between the measurement results by two methods are shown in Fig.8. These are almost within $\pm 5 \mathrm{~mm}$. The standard deviation of the measurement value by this system was about $0.25 \mathrm{~mm}$.

\subsection{Accuracy of Marking}

We carried out the experiment of drawing different types of figures on the ceiling board. The robot given the coordinates of the target point started from arbitrary points. Then it measured self-position and got autonomously to the target point and finally drew specified figures on the ceiling board at the desired point. For example, three marking points were set up as shown in Fig.9, and the drawing figure was square as $500 \mathrm{~mm} \times 500 \mathrm{~mm}$. We measured the coordinates of the drawn square's center and vertex using above mentioned transit in order to investigate the

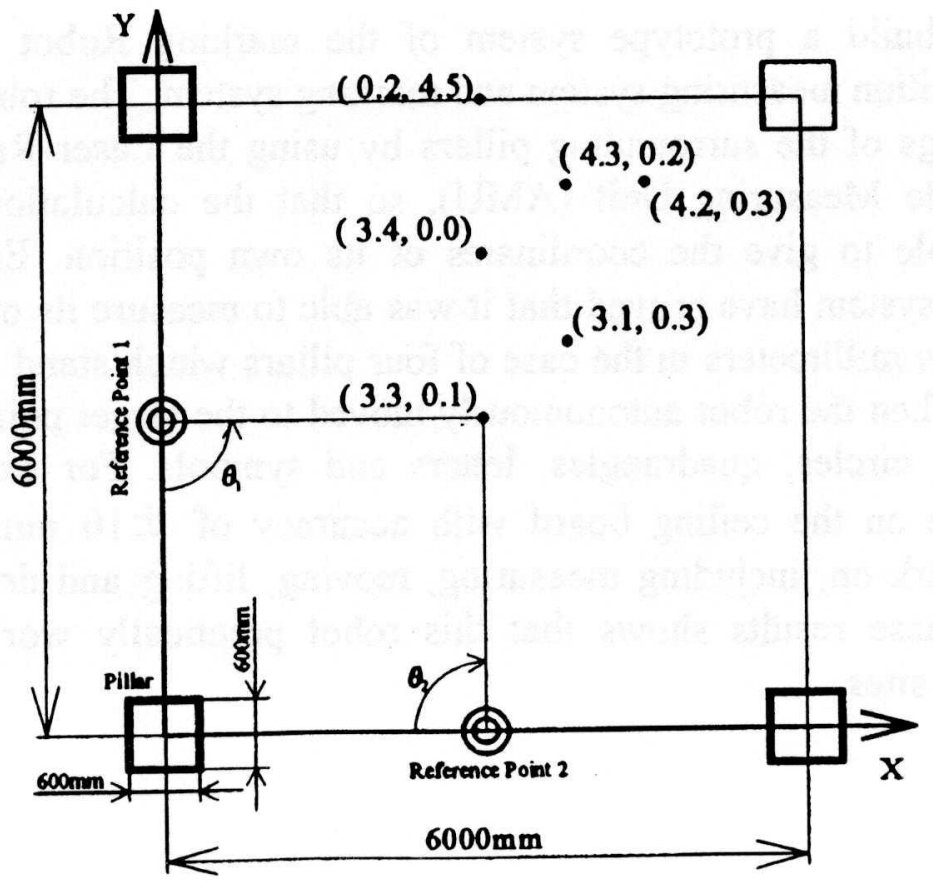

Fig. 8 Measurement result 
marking accuracy. As an example, the result of five test experiments is shown in Fig.10. This figure shows the center coordinates of the square and each vertex and the two sides. The differences between correct figure and drawn figure were within $\pm 10 \mathrm{~mm}$.

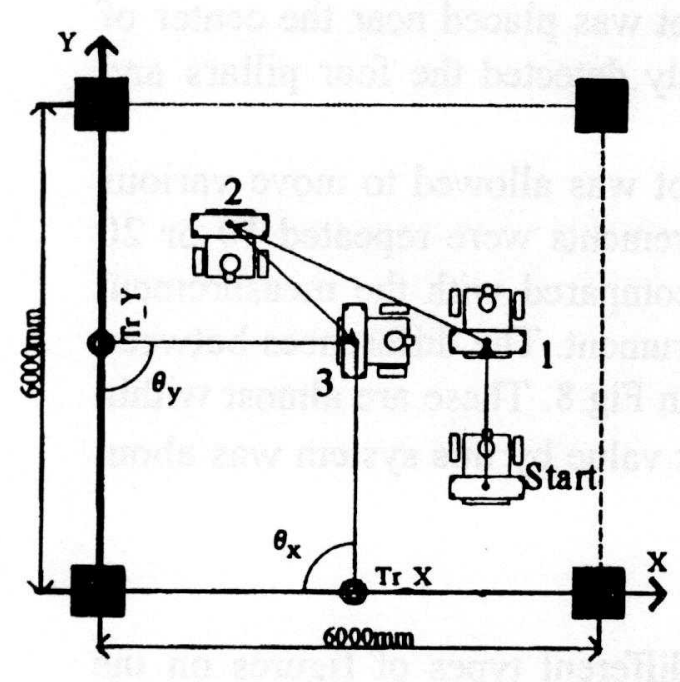

Fig. 9 Marking Position

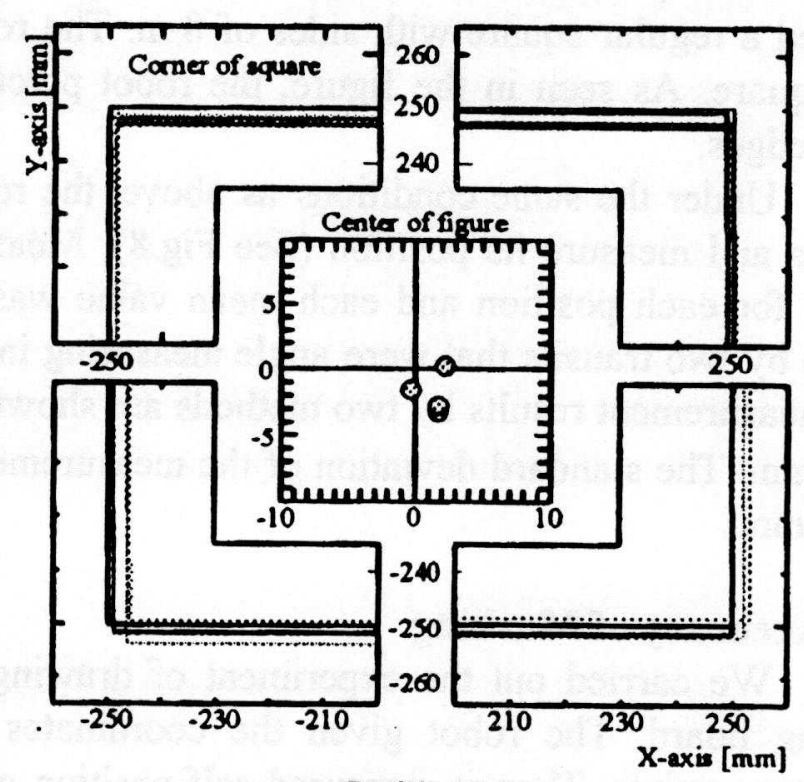

Position 2

Fig. 10 Marking result

\section{Conclusion}

The authors build a prototype system of the marking Robot which was composed of self-position measuring system and marking system. The robot precisely measured the bearings of the surrounding pillars by using the Laser Range Finder (LRF) and the Angle Measuring Unit (AMU), so that the calculation based on triangulation was able to give the coordinates of its own position. Experimental results for prototype system have proved that it was able to measure its own position with an error of a few millimeters in the case of four pillars which stand on the floor an intervals of $8 \mathrm{~m}$. Then the robot autonomously moved to the target point and drew the designated lines, circles, quadrangles, letters and symbols. For example, this robot drew a square on the ceiling board with accuracy of $\pm 10 \mathrm{~mm}$. The time required to put a mark on, including measuring, moving, lifting and drawing, was about 8 minutes. These results shows that this robot practically work at actual building construction sites. 